\title{
EL PENSAMIENTO NARRATIVO EN LA ENSEÑANZA DE LAS CIENCIAS
}

\author{
Agustín Adúriz-Bravo \\ Andrea Revel ChION \\ Universidad de Buenos Aires (UBA), Buenos Aires, Argentina
}

\begin{abstract}
ResUmen: Son aún escasas las investigaciones sobre la importancia del formato narrativo en el aprendizaje de las ciencias; en este artículo reconocemos la necesidad de que se instale este tema en la agenda de trabajo de la didáctica de las ciencias. Retomamos cuestiones planteadas en publicaciones anteriores y esbozamos líneas de indagación promisorias para apuntalar los diversos usos de la narrativa en la educación científica. Examinamos algunas características de las narrativas desde la lingüística y la ciencia cognitiva, reconociendo posibles aportaciones al aula de ciencias: su potencialidad para vehiculizar contenidos conceptuales de la ciencia escolar, su contribución a la comprensión significativa a través de relacionar ideas centrales en una trama, su impacto positivo en la motivación del estudiantado y el aumento de la retención de los aprendizajes debido a su memorabilidad.
\end{abstract}

Palabras-Clave: Narrativas. Pensamiento narrativo. Contextualización. Formación del profesorado de ciencias.

\section{INTRODUCCIÓN}

La didáctica de las ciencias ha comenzado a reconocer -con base en diversas investigaciones hechas en las últimas décadas en campos tales como la lingüística (ADAM; LORDA, 1999) y la ciencia cognitiva (HERMAN, 2007) - que la narrativa es particularmente "afín" al sistema cognitivo humano: 
Es muy probablemente el caso que la manera más natural y más antigua en la que organizamos nuestra experiencia y nuestro conocimiento sea en términos de la forma narrativa. (BRUNER, 1996, p. 121, traducción nuestra)

Por tanto, algunos didactas de las ciencias venimos sugiriendo que sería esencial integrar sistemáticamente las narrativas, cuentos o relatos en la enseñanza de las ciencias en los distintos niveles educativos (ARROIO, 2011; AVRAAMIDOU; OSBORNE, 2008, 2009; HADZIGEORGIOU, 2016; KLASSEN; FROESE-KLASSEN, 2014; LEVINSON, 2006; REVEL CHION; ADÚRIZ-BRAVO, 2014; STRUBE, 1994).

Las narrativas son más sencillamente comprendidas que otros formatos textuales tales como las explicaciones y las argumentaciones, textos "epítome" de la racionalidad lógico-lingüística y hegemónicos en las clases de ciencias. La facilidad para comprender los cuentos o relatos se basa en que la propia experiencia humana se configura en términos de hechos vividos en lugares y tiempos determinados; allí radicaría la potencia de las narrativas producidas al relatar algo que sucede y que va seguido de otros eventos: sus consecuencias (NORRIS et al., 2005).

Precisamente, el éxito de las narrativas en lo que se refiere a su comprensión y recuerdo es lo que las ha convertido en la estructura por excelencia para la transmisión de ideas, prácticas y valores de los que dependió la propia subsistencia de los seres humanos, al menos en los tiempos anteriores a la universalización de la escritura. Generación tras generación, aquellas informaciones vitales que íbamos adquiriendo eran relatadas a los más jóvenes para que fueran atesoradas y vueltas a relatar. De esta manera, un rasgo absolutamente constitutivo de la especie humana sería su capacidad de contar historias (IGARTUA, 2011).

Ahora bien, ¿en qué momento los relatos dejan de formar parte sustancial de la enseñanza de las ciencias? ¿En qué momento el profesorado de ciencias abandona la práctica regular de contar cuentos a sus estudiantes y de hacer que ellos cuenten cuentos? Tal como lo plantea Jay Lemke (1997), la palabra (el habla) es la protagonista privilegiada de las aulas de ciencias, aunque a menudo hegemonizada por los profesores; sin embargo, traspasados los primeros niveles de formación (educación inicial y parte de la primaria), los estudiantes ya no vuelven a escuchar relatos con tanta frecuencia, y los relatos que se narran pasan a ocupar un lugar muy secundario en la estructura de la clase y se vuelven fuertemente estereotipados (constituyendo en su mayoría biografías de científicos de corte "hagiográfico"). En alguna etapa de la escolaridad algo difícil de precisar, el grueso del intercambio lingüístico en 
la enseñanza de las ciencias pasa a constituirse de definiciones, descripciones o explicaciones, generalmente bajo la forma de exposiciones construidas por un único emisor, que es casi siempre el profesor. Pero si esto es así, ¿cómo se reencauzan las dimensiones epistemológica (de construcción de conocimiento) y moral (de transmisión de valores) que Jackson ([1998], 2005) postula para la narrativa?

En esta misma línea cabe preguntarse la razón por la cual el campo de la investigación y la innovación educativa tiende a olvidar el señalamiento hecho por el recientemente fallecido Jerome Bruner (1991) sobre la capacidad de las narrativas para aportar contenidos conceptuales específicos. De acuerdo con ese autor, las narrativas pueden constituirse en "auténticos vehículos de conocimiento" adecuados a diversas disciplinas, muy especialmente a aquellas que modelizan fenómenos con complejidades históricas y causales. Aquí cabe incluir a las ciencias naturales (física, química, biología, etc.), que - a pesar de su clásica preferencia por la dimensión lógico-sintáctica - contienen innumerables ejemplos de "racionalidad narrativa" (IZQUIERDO-AYMERICH, 2014, p. 14-15).

En las secciones que siguen discutiremos esas cuestiones. También recuperaremos algunas potencialidades del formato narrativo para justificar la relevancia de incluirlo de manera protagónica en las clases de ciencias de todos los niveles educativos - desde el inicial hasta el universitario- $y$ en la formación inicial y continuada del profesorado de ciencias. Consecuentemente, sugeriremos que el estudio de las aportaciones de la narrativa a la enseñanza de las ciencias debería cobrar más peso en la agenda de investigación de la didáctica de las ciencias (KLASSEN, 2009).

\section{LAS NARRATIVAS}

Aunque es complejo determinar con univocidad qué constituye una narrativa (DAHLSTROM, 2014; NORRIS et al., 2005; PALAPANIDI, 2013;), seguimos aquí a Labov y Waletzky (1967), quienes la definen -con gran economía formal- como cualquier secuencia de oraciones ("clauses") que contenga al menos un conector temporal ("temporal juncture"); tal conector establece la sucesión entre dos o más de las oraciones del texto completo. Para clarificar ideas, revisemos uno de los ejemplos más sencillos estudiado por estos autores: "Conozco a un chico llamado Harry. Otro chico le tiró una botella directo a la cabeza y le tuvieron que dar siete puntos de sutura" (LABOV; 
WALETZKY, 1967, p. 10. traducción nuestra). Según ellos, se trata de una genuina narrativa porque hay clara indicación de una conexión temporal entre "otro chico le tiró una botella directo a la cabeza" y "le tuvieron que dar siete puntos de sutura". Esta es para Bruner (1991) la propiedad más importante del formato narrativo: ser inherentemente secuencial; tal característica permite - al receptor del relato - aprehender la trama y, al mismo tiempo, entender los distintos componentes puestos en relación con dicha trama. Se hace así comprensible y recordable la idea general.

Uno de los rasgos centrales de las narrativas es el enorme poder de atracción que tienen sobre la imaginación humana, lo que ha sido denominado "efecto narrativo" (BRUNER, 1991; NORRIS et al., 2005) y parece ser el responsable de su memorabilidad. Tal como nos dice Jackson ([1998], 2005), los buenos relatos se recuerdan. Esta es una primera razón de peso para su inclusión en la enseñanza de las ciencias, ya que los contenidos científicos que forman parte integral de una narrativa pueden quedar "disponibles" como insumos para ser recuperados y puestos al servicio de resolver problemas o producir explicaciones. Así, en las aulas de ciencias se podrían establecer unas relaciones "fructíferas", de mutuo apoyo, entre los relatos y la línea principal de discurso de la clase, incluyendo aquí la argumentación científica escolar (REVEL CHION, 2014).

Bruner (2002) sugiere que el cuento o relato es un medio para comprender y hacer comprender, en el cual el usuario no es capaz de identificar fehacientemente por qué entiende más y mejor a partir de él, pues

nuestras intuiciones sobre cómo hacer una historia o cómo entenderla correctamente son tan implícitas, nos resultan tan inaccesibles, que fracasamos cuando tratamos de explicarnos a nosotros mismos, o a alguien poco convencido, qué hace de algo una historia [...] (BRUNER, 2002, p. 3-4. traducción nuestra).

Sin embargo, creemos que sí se debería enseñar explícitamente a los profesores de ciencias cuáles son algunas de las características de las narrativas que las convierten en insumos apropiados para la enseñanza. Estas características advienen de la interacción entre ese formato textual y el sistema cognitivo humano.

En efecto, Avraamidou y Osborne (2009) sugieren que la cadena de eventos bien conectados entre sí, la estructura global signada por un inicio, un desarrollo y un final ordenados temporalmente y relatados en tiempo pasado, y la presencia de "agentes" - que se corresponden ya sea con 
actores, ya con "entidades" no humanas que hacen algo - son los principales elementos de la narrativa que hacen posible la comprensión y el recuerdo, merced a que "resuenan" con los modos de almacenamiento y procesamiento de información evolutivamente seleccionados en los humanos. Los autores resaltan así las grandes diferencias entre las narrativas y los textos expositivos, con los que más frecuentemente entran en contacto los estudiantes de ciencias. Las exposiciones - y otros tipos textuales científicos estándar carecen del "triunvirato" narrativo clásico (DAHLSTROM, 2014): no exhiben secuencialidad, ni intencionalidad ("agencia" con personajes), y tienen unos modos de causalidad limitados (mecanísticos). Estas carencias serían precisamente las responsables de la mayor dificultad para su comprensión.

Los mismos Avraamidou y Osborne (2009) argumentan que el propósito de las narrativas no sería solamente el de lograr la comprensión de la esfera de lo humano, sino que también podrían colaborar en nuestra explicación del mundo natural. En este sentido, indican que la enseñanza de las ciencias, que pretende construir colectivamente en el aula explicaciones causales, plausibles y consistentes sobre los hechos del mundo, podría verse robustecida por medio del uso del formato narrativo.

Otra característica distintiva de las narrativas es que su estructura está atravesada por la presencia de miradas personales, psicológicas, históricas, culturales, ideológicas, políticas, económicas, etc., de parte de y acerca de los agentes incluidos en la narración; esta riqueza de miradas integradas en el relato impactaría muy positivamente tanto en la motivación del receptor como en la memorabilidad del texto y en el establecimiento de conexiones complejas entre las partes. No resultan desconocidos para el profesorado la atención y el interés que provoca en los estudiantes cualquier breve alusión a las vivencias personales o motivaciones de los personajes cuyas teorías se enseñan en las clases de ciencias - no muy diferente de lo que sucede en los niños y niñas más pequeños cuando sus maestros o padres les cuentan historias.

En el ámbito de la educación científica, al interés y la significatividad que las narrativas generan en los estudiantes se suma que ellas contribuyen a construir una "imagen de ciencia" fuertemente contextualizada: humana, cambiante, históricamente situada, atravesada por intereses y valores; tal imagen se adecua a las perspectivas epistemológicas actuales (ADÚRIZBRAVO; ARIZA, 2013; REVEL CHION; ADÚRIZ-BRAVO, 2014). Así, las narrativas serían un vehículo potente para enseñar sobre la llamada naturaleza de la ciencia (ARROIO, 2011; ADÚRIZ-BRAVO, 2014). 
Por otra parte, Stinner et al. (2003) alertan acerca de la distancia existente entre las exposiciones que los profesores aportan en las clases de ciencias y los conocimientos de partida con que cuentan los estudiantes (entre ellos, las "ideas previas"). Asumiendo que esa distancia es usualmente grande, sugieren que los nuevos contenidos científicos escolares "duros" se introduzcan también en formato narrativo, contextualizándolos; así se podrían generar aprendizajes más significativos.

Con ese mismo espíritu, Stephen Klassen (2007) sugiere que la contextualización del conocimiento científico con narrativas requiere siempre de cinco elementos; haremos aquí nuestra reinterpretación de la propuesta original del autor. Habría primeramente un elemento teórico y un elemento práctico-experimental, que permitirían abordar las preguntas e hipótesis de las investigaciones narradas, relacionándo con las ideas compartidas por la comunidad científica y con las intervenciones que se pueden hacer sobre el mundo. Luego tendríamos el elemento social y el elemento histórico, que enmarcarían la narrativa, indicando el tiempo y el lugar en que transcurre, cargándola de significados y valores culturales compartidos. Por último necesitaríamos un elemento afectivo, constituido por las vivencias y sentimientos que los agentes del relato exponen o experimentan (por ejemplo, entrega, irresponsabilidad, confusión, solidaridad, desesperanza, envidia, satisfacción, etc.) y que a su vez evocan y movilizan emociones en los oyentes/lectores.

Para Klassen (2007), esos cinco elementos del contexto "orientados por el relato" ("story-driven") interactúan y se retroalimentan dándole vida, razón por la cual se impone un tratamiento conjunto de todos ellos en la clase de ciencias. Ello se puede hacer, por ejemplo, introduciendo narrativas ricas basadas en la historia de la ciencia (METZ et al., 2007).

\section{LA NARRATIVA ENTENDIDA COMO “MODO" DE PENSAMIENTO}

En la sección anterior afirmamos que las razones por las cuales las narrativas se muestran "amigables" a la cognición humana tienen que ver con aquellas características del formato que se adecuan a cierta racionalidad para la que estamos naturalmente preparados. Así, según algunas corrientes del campo de la psicología cognitiva, la narración no se reduce a un tipo de discurso, sino que también es una forma de pensamiento, un modo por medio del cual los sujetos son capaces de organizar (dar sentido) y almacenar 
(tener disponible) su propia experiencia. En esta línea, la narración constituiría un auténtico modo de funcionamiento cognitivo (SANTAMARÍA SANTIGOSA; RAMÍREZ, 1998). Se podría hablar así - como lo hace Bruner (1991) - de "pensamiento narrativo" ("narrative thought"): a través de él, la mente humana crearía significados originales en su interacción con el mundo. Las operaciones fundamentales de construcción de sentido que corresponden al pensamiento narrativo serían propias tanto de los individuos como de la humanidad en su conjunto (LODGE, 2002).

Santamaría Santigosa y Ramírez (1998) recogen la idea de que tradicionalmente se han considerado en el ser humano dos vías dicotómicas para organizar experiencias y construir significados; Bruner (1986) incluso da a estas vías el estatus ontológico de "clases naturales" ("natural kinds"). Una de ellas, fuertemente lógica y formal, propia de las disciplinas científicas en su forma final, axiomatizada, es llamada por Bruner (1986)"modo paradigmático". Este modo
intenta cumplir el ideal de un sistema formal, matemático, de descripción y explicación. Emplea la categorización o conceptualización y las operaciones mediante las cuales las categorías se establecen, ejemplifican, idealizan y relacionan entre sí para formar un sistema (BRUNER, 1986, p. 12. traducción nuestra)

La segunda vía sería el "modo sintagmático" de Bruner (1986), por medio del cual los sujetos - en contexto - interactúan entre sí, crean significados y los narran. Este modo narrativo sería más afín al sentido común; es por ello que, como señalan muchos autores, se ha tendido a creer que la narración - en tanto que una forma natural, intuitiva - es menos elaborada y reflexiva, y por tanto de estatus más bajo, que el pensamiento lógico. Se trataría de un pensamiento "inmaduro", que aparece tempranamente en el desarrollo, y que se espera sea reemplazado en su debido momento por una forma de pensar lógica, abstracta y racional. De esta creencia probablemente proviene la progresiva pérdida de peso de las narrativas en la enseñanza de las ciencias: el profesorado podría considerar que ellas no consiguen transmitir el raciocinio científico en sus formas más elaboradas.

Ahora bien, con base en lo que hemos venido diciendo, podríamos ver los dos "modos de pensamiento" a la Bruner como "irreducibles" pero "complementarios": muy diferentes entre sí, pero igualmente válidos para significar la experiencia humana en general y obtener comprensión científica del mundo en particular. Así, podrían coexistir sin mayores dificultades 
en las clases de ciencias, aportando cada uno sus fortalezas (IZQUIERDOAYMERICH, 2014). Mientras que el pensamiento paradigmático haría posible emitir argumentos, es decir, "verdades" con relaciones lógicas entre las proposiciones, el pensamiento narrativo supondría otro tipo de ligazón entre sus ideas: las relaciones de temporalidad y secuencialidad que introdujimos en la sección anterior.

Adherimos aquí al planteo de Bruner (1996) de que, mientras los argumentos que se elaboran desde el modo paradigmático convencen por su verdad, los relatos del modo narrativo convencen por su semejanza con la vida, su verosimilitud. Su enorme valor para las clases de ciencias estribaría en que nos permiten construir explicaciones particulares y "sensibles al contexto" ("context-sensitive": HADZIGEORGIOU, 2016). Insistimos entonces en la propuesta de que este modo acompañe la clase de ciencias usual, basada en las leyes abstractas y universales consustanciales al modo paradigmático:

La formación académica "deductiva" intenta utilizar una racionalidad lógica en la enseñanza, la racionalidad lógica de las teorías ya formalizadas; el problema es que, aun en el caso de que los alumnos estén dispuestos a incorporar [los] axiomas [...] conectan con dificultad con los fenómenos, y la historia [de la ciencia] nos muestra por qué. La actividad humana "competente" requiere también "racionalidad narrativa": [l]a emergencia de conocimiento se produce en contextos específicos en los cuales la actividad es genuina. La historia de las ciencias ofrece posibilidades de identificarlos (IZQUIERDO-AYMERICH, 2014, p. 14-15).

Proponemos aquí, como lo han hecho antes otros autores (GÓMEZ GALINDO, 2013; NORRIS et al., 2005), que una puerta de entrada para integrar el modo paradigmático y el modo sintagmático en las clases de ciencias estribaría en tomar el modelo de la explicación como unificación, del filósofo de la ciencia inglés Philip Kitcher (1989). Para Kitcher, el valor de una explicación científica reside en la capacidad que tiene de permitir a quienes la formulan la organización altamente económica y estructurada del conocimiento:

Nuestras explicaciones encajan los fenómenos en una cosmovisión general que tiene unas pocas suposiciones independientes. [L]a visión de unificación de la explicación requiere que las explicaciones sean evaluadas en relación con un "almacén de explicaciones" ["explanatory store"] [...] disponible en un determinado momento. [...] La comprensión aumenta [...] a medida que el número de hechos que tenemos que tomar en bruto se reduce. El conocimiento científico es de esta manera unificado en la medida en 
que podemos derivar el mayor número de hechos del menor número de suposiciones (NORRIS et al., 2005, p. 548. traducción nuestra).

La noción kitcheriana del "almacén" de explicaciones estrechamente relacionadas entre sí y conteniendo un conjunto pequeño y conexo de ideas que pueden ser empleadas repetidamente para derivar diversos hechos "satisfactoriamente explicados" nos permite postular un papel distintivo para la narrativa. Ella proveería la secuencia de explicaciones organizadas y contextualizadas.

Muchos de los autores que hemos venido citando a lo largo de este artículo reconocen un "giro narrativo" en la segunda mitad del siglo XX: el interés de los estudios sociales se redirige hacia entender a fondo el papel que juega la narrativa en la vida humana. Pero, como afirma el pedagogo griego Yanis Hadzigeorgiou (2016),

si se ha de redescubrir la narrativa, como de hecho ha sucedido en las últimas dos décadas, entonces se deberá poner mucha más atención a las nociones de oralidad y de contar cuentos [storytelling] y a sus consecuencias para la enseñanza y el aprendizaje y también para la organización del currículo (HADZIGEORGIOU, 2016, p. 84. traducción nuestra).

Ello implica asentar una agenda de trabajo - ya tímidamente iniciada - para la didáctica de las ciencias que incluya investigar sobre la naturaleza del pensamiento narrativo, su función en la construcción del discurso científico y su valor en una educación científica de calidad para todos. También nos parece central realizar investigación empírica acerca de qué relatos son los más adecuados para el aula de ciencias y cómo se han de construir, implementary evaluar (METZ et al., 2007). Por último, encontramos necesario profundizar en la discusión acerca de las aportaciones de la narrativa a la profesionalización del profesorado de ciencias.

\section{A MODO DE CONCLUSIÓN}

En este artículo partimos de convenir con el diagnóstico de que la narrativa es "un medio subutilizado para aprender" (EGAN, 1986, p. 5. traducción nuestra) en el ámbito de la educación científica; por ello hemos intentado señalar que la escasez de buenos relatos en la clase de ciencias tiene consecuencias negativas para: 1 . la retención a mediano plazo de 
los aprendizajes; 2. la capacidad de usar lo aprendido en situaciones problemáticas genuinas o de transferirlo a nuevos contextos; 3 . la motivación y el compromiso del estudiantado con la ciencia; 4 . la habilidad para manejar explicaciones multicausales, genéticas, funcionales o teleológicas; y 5 . la construcción de una imagen de ciencia como actividad humana que tiene lugar inmersa en un contexto social, imagen que, tal como sugiere Lemke (1997), debería primar en la enseñanza de las ciencias.

Así, hemos apuntado algunos aportes que puede hacer el formato narrativo para el aprendizaje de los contenidos científicos escolares, poniendo en relación esos aportes con las principales características del pensamiento narrativo. Creemos que esos aportes serían especialmente importantes en el caso de las problemáticas interdisciplinarias y los asuntos sociocientíficos, que entraman las teorías clásicamente enseñadas con contenidos y enfoques de otras áreas y que, por tanto, exhiben mucha mayor complejidad para su manejo solvente.

En el caso específico de la formación del profesorado de ciencias, a todas las ventajas ya expuestas a lo largo de este trabajo se sumarían las de proveer metodologías específicas para la tarea docente, modelar valores acerca del enseñar, ayudar al profesorado novel a construir conocimiento profesional, facilitar puertas de acceso a la presentación de contenidos multidisciplinares y proporcionar un cuerpo robusto de nociones de naturaleza de la ciencia que serán objeto de enseñanza (HADZIGEORGIOU, 2016; KLASSEN; FROESE-KLASSEN, 2014; REVEL CHION; MEINARDI; ADÚRIZ-BRAVO, 2013).

\section{NARRATIVE THOUGHT IN SCIENCE EDUCATION}

ABSTRACT: There is still very little research on the contributions of the narrative format to science learning. In this paper, we recognise the need to include this issue in the agenda of science education as a discipline. We discuss some questions available in the literature and we sketch research lines that we consider fruitful to support the uses of narrative in science teaching. We examine some characteristics of narratives from the perspective of linguistics and cognitive science, and we identify possible contributions to science classes: their potential to convey conceptual content of school science, their help to meaningful 
understanding through relating central ideas in a plot, their positive impact in students' motivation, and the improvement of retention due to its memorability. KEYWORDS: Narratives. Narrative thinking. Contextualisation. Science teacher education.

\section{O PENSAMENTO NARRATIVO NO ENSINO DE CIÊNCIAS}

RESUMO: São ainda raras as pesquisas a respeito da importância do formato narrativo na aprendizagem de ciências. Neste artigo reconhecemos a necessidade de inserir este tema na agenda de trabalho da didática do ensino de ciências. Retomamos algumas questões colocadas em publicações anteriores e esboçamos linhas de pesquisa que consideramos promissoras para fortalecer os diversos usos da narrativa na educação científica. Examinamos algumas características das narrativas, a partir da linguística e da ciência cognitiva, reconhecendo seus possíveis aportes às aulas de ciências: o potencial para veicular conteúdos conceituais da ciência escolar, sua contribuição para o entendimento significativo por meio do relacionamento de ideias centrais numa trama, seu impacto positivo na motivação dos estudantes, e o aumento da retenção da aprendizagem, dada a memorabilidade.

Palavras-chave: Narrativas. Pensamento narrativo. Contextualização. Formação dos professores de ciências.

\section{REFERENCIAS}

ADAM, J. M.; LORDA, C. U. Lingüística de los textos narrativos. Barcelona: Ariel, 1999.

ADÚRIZ-BRAVO, A. Teaching the nature of science with scientific narratives. Interchange, v. 45, n. 3, p. 167-184, 2014.

ADÚRIZ-BRAVO, A.; ARIZA, Y. Las imágenes de ciencia y de científico: Una puerta de entrada a la naturaleza de la ciencia. In: ADÚRIZ-BRAVO, A.; DIBARBOURE, M.; ITHURRALDE, S. (Org.). El quehacer del científico al aula: Pistas para pensar. Montevideo: Fondo Editorial Queduca, 2013. p. 13-20.

ARROIO, A. Cinema as narrative to teach nature of science in science education. Western Anatolia Journal of Educational Sciences, número especial, p. 87-92, 2011. Disponível em: <http://webb.deu.edu.tr/baed/giris/baed/ozel_sayi/87-92.pdf>. Acesso em: 20 jun. 2016.

AVRAAMIDOU, L.; OSBORNE, J. F. Science as narrative: The story of the discovery of penicillin. The Pantaneto Forum, n. 31, s/p., 2008. Disponível em: <http://www. pantaneto.co.uk/issue31/avraamidou.htm>. Acesso em: 20 jun. 2016. 
AVRAAMIDOU, L.; OSBORNE, J. F. The role of narrative in science education. International Journal of Science Education, v. 31, n. 4, p. 1-25, 2009.

BRUNER, J. S. Actual minds, possible worlds. Cambridge: Harvard University Press, 1986. . Actos de significado: Más allá de la revolución cognitiva. Madrid: Alianza, 1991.

. The culture of education. Cambridge: Harvard University Press, 1996.

Making stories: Law, literature, life. Nueva York: Farrar, Straus and Giroux, 2002.

DAHLSTROM, M. F. Using narratives and storytelling to communicate science with nonexpert audiences. Proceedings of the National Academy of Science, n. 111, suppl. 4, p. 13614-13620, 2014. Disponível em: <http://www.ncbi.nlm.nih.gov/pmc/ articles/PMC4183170/>. Acesso em: 20 jun. 2016.

EGAN, K. Teaching as story-telling. Chicago: University of Chicago Press, 1986.

GÓMEZ GALINDO, A. A. Explicaciones narrativas y modelización en la enseñanza de la biología. Enseñanza de las Ciencias, v. 31, n. 1, p. 11-28, 2013.

HADZIGEORGIOU, Y. Imaginative science education: The central role of imagination in science education. Dordrecht: Springer, 2016. p. 83-120.

HERMAN, D. Storytelling and the sciences of mind: Cognitive narratology, discursive psychology, and narratives in face-to-face interaction. Narrative, n. 15, p. 306-334, 2007.

IGARTUA, J. J. Mejor convencer entreteniendo: Comunicación para la salud y persuasión narrativa. Revista de Comunicación y Salud, v. 1, n. 1, p. 79-83, 2011.

IZQUIERDO-AYMERICH, M. Pasado y presente de la química: Su función didáctica. In: MERINO, C.; ARELLANO, M.; ADÚRIZ-BRAVO, A. (Org.). Avances en didáctica de la química: Modelos y lenguajes. Valparaíso: Ediciones Universitarias de Valparaíso, 2014. p. 12-36.

JACKSON, P (1998). Sobre el lugar de la narrativa en la enseñanza. In: McEWAN, H.; EGAN, K. (Org.). La narrativa en la enseñanza, el aprendizaje y la investigación. Buenos Aires: Amorrortu, 2015. p. 25-51.

KITCHER, P. Explanatory unification and the causal structure of the world. In: KITCHER, P.; SALMON, W.C. (Org.). Minnesota studies in the philosophy of science: Vol. XIII: Scientific explanation. Minneapolis: University of Minnesota Press, 1989. p. 410-499.

KLASSEN, S. The application of historical narrative in science learning: The Atlantic cable story. Science \& Education, n. 16, issue 3, p. 335-352, 2007.

. The construction and analysis of a science story: A proposed methodology. Science \& Education, v. 18, n. 3, p. 401-423, 2009. 
$\therefore$ FROESE-KLASSEN, C. Science teaching with historically based stories: Theoretical and practical perspectives. In: Matthews, M. (Org.). International handbook of research in history and philosophy for science and mathematics education. Dordrecht: Springer, 2014. p. 1503-1529.

LABOV, W.; WALETZKY, J. Narrative analysis: Oral version of personal experience. Journal of Narrative and Life History, v. 7, n. 1-4, p. 3-38, 1967.

LEMKE, J. Aprender a hablar ciencia: Lenguaje, aprendizaje y valores. Barcelona: Paidós, 1997. Título original: Talking Science: Language, learning and values.

LEVINSON, R. The use of narrative in supporting the teaching of socio-scientific issues: A study of teachers' reflections. Interacções, v. 2, n. 4, p. 24-41, 2006. Disponível em: <http://revistas.rcaap.pt/interaccoes/article/view/319/275>. Acesso em: 20 jun. 2016.

LODGE, D. El arte de la ficción. Barcelona: Península, 2002. Título original: The Art of Fiction.

METZ, D. et al. Building a foundation for the use of historical narratives. Science \& Education, n. 16, issue 3, p. 313-334, 2007.

NORRIS, S.P. et al. A theoretical framework for narrative explanation in science. Science Education, v. 89, n. 4, p. 535-563, 2005.

PALAPANIDI, K. Narración y argumentación: Dos tipos discursivos, dos modos de pensamiento. redELE: Revista Electrónica de Didáctica del Español Lengua Extranjera, n. 25, s/p., 2013. Disponível em: <http://www.mecd.gob.es/dctm/redele/MaterialRedEle/Revista/2013/2013_redELE_25_22Palapanidi.pdf?documentld=0901e72b81 74393c>. Acesso em: 20 jun. 2016.

REVEL CHION, A. Narrativas y argumentación: Unas relaciones fructíferas para la enseñanza de la salud. Ponencia presentada en el XI JORNADAS NACIONALES Y VI CONGRESO INTERNACIONAL DE ENSEÑANZA DE BIOLOGÍA, Gral. Roca, Argentina, 2014.

.; MEINARDI, E.; ADÚRIZ- BRAVO, A. El formato narrativo en la enseñanza de un modelo complejo de salud y enfermedad. Revista de Educación en Biología, v. 16, n. 1, p. 28-36, 2013.

; ADÚRIZ-BRAVO, A. ¿Qué historias contar sobre la emergencia de enfermedades? Tecné, Episteme y Didaxis, n. 36, p. 47-59, 2014.

SANTAMARÍA SANTIGOSA, A.; RAMÍREZ, J. La narración: Un modo de pensamiento. In: JORNADAS DE PSICOLOGÍA DEL PENSAMIENTO. Santiago de Compostela: Universidad de Santiago de Compostela, 1998. p. 247-259.

STINNER, A. et al. The renewal of cases studies in science education. Science \& Education, v. 12, issue 7, p. 617-643, 2003.

STRUBE, P. Narrative in the science curriculum. Research in Science Education, v. 24, n. 1, p. 313-321, 1994. 
Agustín Adúriz-Bravo: Docente-Investigador en el Instituto CeFIEC de la Universidad de Buenos Aires, Argentina. Especialista en didáctica de las ciencias naturales.

E-mail: aadurizbravo@cefiec.fcen.uba.ar

Andrea Revel Chion: Jefe de Trabajos Prácticos Regular con Dedicación Exclusiva en el Instituto CeFIEC de la Universidad de Buenos Aires. Especialista en didáctica de las ciencias naturales. Líneas de trabajo: argumentación científica escolar y uso de las narrativas en la enseñanza de las ciencias.

E-mail: andrearevelchion@yahoo.com.ar 